\title{
Tecnologías de la información y comunicación como estrategia pedagógica para la convivencia escolar ${ }^{1}$
}

\section{Information and communication technologies as a pedagogical strategy for school coexistence}

DOI: http://dx.doi.org/10.17981/cultedusoc.9.1.2018.08

Fecha de recepción: 11/04/2018. Fecha de aceptación: 10/07/2018

Jeannette del Pilar Florián-Rodríguez ${ }^{2}$ iD

Yacqueline Espejo-Rovira; Melvis Marolis Martínez-Márquez; Adelina Esther Herrera-Morales; Josefa María Castillo-De Aguas; Neirith Mercedes Fuentes-Tritón; Henry Parra-Suarez; Karina Vianeth Mendoza-Montecino; Sandy Paola Tapia ${ }^{3}$

Para citar este artículo

Florián-Rodríguez, J., Espejo-Rovira, Y., Martínez-Márquez, M., Herrera-Morales, A., Castillo-De Aguas, J., Fuentes-Tritón, N., Parra-Suarez, H., Mendoza-Montecino, K. y Tapia, S. (2018). Tecnologías de la información y comunicación como estrategia pedagógica para la convivencia escolar. Cultura. Educación y Sociedad 9(1), 108-120. DOI: http://dx.doi.org/10.17981/cultedusoc.9.1.2018.08

\section{Resumen}

El avance en las tecnologías de la información y la comunicación ha revolucionado todos los campos de la actividad humana, exigiendo a las instituciones educativas diseñar e implementar modelos de enseñanza - aprendizaje, fortaleciendo las competencias de los estudiantes mediante recursos tecnológicos. El propósito de la presente investigación es utilizar las TIC como estrategia pedagógica para la convivencia escolar. El estudio es de tipo cualitativo utilizando el modelo de investigación acción, la población estuvo conformada por ciento veintiséis (126) estudiantes de la Institución Educativa Departamental Humberto Velásquez García, sede Cristo Rey. Para recoger los datos se utilizaron, relatorías individuales, institucionales y diario de campo. Se concluye que mediante ambientes de aprendizaje apoyados con herramientas TIC se potencian los aprendizajes, formación ciudadana y convivencia escolar. Por tal motivo se diseñó una plataforma virtual con estrategias de comunicación apoyadas en las TIC, utilizada para generar cambios en las relaciones escolares de los alumnos.

Palabras clave: tecnologías de la información y comunicación, estrategia pedagógica, convivencia escolar.

\begin{abstract}
Advances in information and communication technologies have revolutionized all fields of human activity, requiring educational institutions to design and implement teaching - learning models, strengthening students' competences through technological resources. The purpose of this research is to use ICT as a pedagogical strategy for school coexistence. The study is of qualitative type using the action research model, the population consisted of one hundred and twenty-six (126) students of the Humberto Velasquez Garcia Departmental Educational Institution, Cristo Rey campus. In order to collect the data, individual, institutional and field diary reports were used. It is concluded that through learning environments supported by ICT tools, learning, citizenship training and school coexistence are enhanced. For this reason, a virtual platform was designed with communication strategies supported by ICT, used to generate changes in students' school relationships.
\end{abstract}

Keywords: technologies of information and communication, pedagogical strategy, school cohabitation.

\footnotetext{
${ }^{1}$ Este artículo ha sido derivado del Programa de Fortalecimiento de la Cultura Ciudadana y Democrática CT+I a través de la IEP apoyada en TIC en el Departamento de Magdalena: CICLON

${ }^{2}$ Docente de la Institución Educativa Humberto Velázquez García y líder del grupo de investigación Innovadores de la Educación. Correo de correspondencia: piliflorian@hotmail.com

${ }^{3}$ Docentes de la Institución Educativa Humberto Velázquez García, Sede Cristo Rey y miembros del grupo de investigación Innovadores de la Educación.
}

- The author; licensee Universidad de la Costa - CUC. 


\section{Introducción}

Los cambios socioculturales, la globalización, la sociedad del conocimiento y la información y las capacidades intelectuales que posee el estudiante de hoy, han conllevado a reflexionar e incluso modificar el paradigma educacional tradicional y ajustarlo a las nuevas demandas sociales, el docente unos de los actores principales de este proceso también debe transformar sus prácticas mejorando su quehacer pedagógico, modificando su perfil y competencias, innovando sus metodologías y estrategias de enseñanza. Es conveniente evaluar constantemente qué estrategias de enseñanza se están desarrollando en el aula, si son coherentes con las necesidades y estilos de aprendizaje de los estudiantes, llevándolos a ser el protagonista y responsable de su aprendizaje. Es importante destacar, que el estudiante de hoy es un "nativo digital".

Igualmente el impacto que ha generado y está generando las tecnologías educativas ha obligado su inmediata incorporación por su triple función: como instrumento facilitador los procesos de aprendizaje (fuente de información, canal de comunicación entre formadores y estudiantes, recurso didáctico), como herramienta para el proceso de la información y como contenido implícito de aprendizaje (los estudiantes al utilizar las TIC aprenden sobre ellas, aumentando sus competencias digitales), siendo ineludible para el docente actual el empleo de estrategias de enseñanza innovadoras y ajustables a los recursos tecnológicos disponibles en la Institución.

Las instituciones educativas debido a los avances tecnológicos, han tenido la necesidad de integrar las tecnologías de la información y la comunicación (TIC) al currículo, sin embargo, en las zonas rurales del país aún existen limitaciones para llevar a cabo este proceso, que van desde la accesibilidad, hasta el uso adecuado de los recursos tecnológicos, por tal razón el grupo de investigación tuvo como objetivo principal utilizar las TIC como estrategia pedagógica para la convivencia escolar, identificando qué metodologías son las más apropiadas en el entorno social de la institución, como adaptarlas al currículo y a las necesidades de los educandos. Logrando con ello propiciar cambios en la organización escolar la cual tiene arraigados métodos de enseñanza tradicionalistas.

En este panorama, las TIC juegan un papel clave, ofreciendo nuevos contextos y posibilidades para el desarrollo de dichas competencias. Las nuevas tecnologías generan espacios de comunicación idóneos para la formación de profesionales dentro de modalidades educativas completamente a distancia o híbridas. La incorporación de las nuevas tecnologías ha puesto de manifiesto la necesidad de que los estudiantes aprendan a comunicarse mediante distintas herramientas. Específica-mente, la argumentación en la formación universitaria en línea ha sido objeto de estudio por sus implicaciones para comunicar ideas, adquirir conocimiento, desarrollar un pensamiento científico y crítico, además de generar conocimiento para enfrentar problemas complejos.

Además, el Ministerio de Educación Nacional de Colombia ha implementado una ruta de apropiación de TIC para el Desarrollo Profesional Docente en el uso e incorporación de este recurso en el apoyo de los diseños curriculares (uso pedagógico) e innovar y transformar las prácticas educativas. También ha brindado espacios en redes de acompañamiento, evaluación y aprendizaje, así como banco 
de experiencias y recursos educativos en todas las áreas del saber en los diferentes portales educativos nacionales.

Políticas nacionales que buscan afianzar el uso responsable, eficiente y productivo de las TIC, así como incorporar estas tecnologías en los procesos pedagógicos como un eje estratégico para mejorar la calidad y el desarrollo de las competencias básicas y laborales propiciando escenarios educativos dinámicos y motivadores que faciliten el aprendizaje significativo de los estudiantes, pero aunque existen las condiciones que favorezcan la incursión del uso pedagógico de las TIC en el aula, se continúan en algunos casos con las mismas metodologías, estrategias, recursos o materiales de apoyo.

La institución educativa Humberto Velázquez García en su sede Cristo Rey ha venido presentando debilidades en materia tecnológica, además, los estudiantes manifiestan conductas disruptivas que han generado altos índices de indisciplina, lo cual ha sido desfavorable a nivel de convivencia escolar, por lo anterior, mediante la investigación se buscó fomentar una cultura ciudadana de Ciencia, Tecnología e Innovación en la población infantil y juvenil, a través de las TIC, ofreciendo a la comunidad científica, educativa y a la sociedad en general, planteamientos, métodos y procesos encaminados a lograr una eficiente organización que puedan ser integrados al currículo o al PEI.

Por otro lado, la constitución política de Colombia, ha sido denominada en ambientes académicos como "la constitución incluyente", puesto que su perspectiva social, económica y cultural, posibilitará herramientas que permitirá visibilizara a actores sociales que históricamente no habían sido reconocidos, así, en su artículo 7 manifiesta que: "El Estado reconoce y protege la diversidad étnica y cultural de la Nación Colombiana". Afirmando en el artículo 13 que "Todas las personas nacen libres e iguales ante la ley, recibirán la misma protección y trato de las autoridades y gozarán de los mismos derechos, libertades y oportunidades sin ninguna discriminación por razones de sexo, raza, origen nacional o familiar, lengua, religión, opinión política o filosófica. El Estado promoverá las condiciones para que la igualdad sea real y efectiva, adoptando medidas a favor de grupos discriminados o marginados".

En las sociedades actuales las tecnologías de la información y la comunicación impregnan todos los quehaceres de la vida en las personas, de ahí la importancia que cobran los procesos de alfabetización tanto informacional como digital que provea de herramientas necesarias tanto para el procesamiento de la información como para el manejo técnico de la misma, lo que lleva a plantear un modelo educativo que contemple las diferentes dimensiones: tecnológica, pedagógica $\mathrm{y}$ administrativa en los procesos de formación, (Avalos, 2008).

Garrell (2009), explica que el docente cumple un rol de mediador en la convivencia escolar el cual debe fomentar un clima favorable en la escuela, que permita buscar diferentes alternativas de solución para invitar a los estudiantes a reflexionar sobre sus ideas y comportamientos propiciando así una convivencia en armonía. Para ello se sugiere la aplicación de una educación en que promueva dialogo, participación, pensamiento crítico, discusión e integración.

Ante la necesidad de ofrecer escenarios motivadores e innovadores con uso de TIC para propiciar aprendizajes significativos se requiere utilizar las TIC como estrategia pedagógica para la convivencia 
escolar para tomar medidas de acción que ofrezcan a los docentes el direccionamiento en su quehacer pedagógico y enfocarlo en elevar el nivel académico de la institución. La investigación permitirá, además, valorar si los recursos disponibles en la institución son pertinentes, si los docentes poseen competencias en TIC necesarias que conduzca la implementación de metodologías innovadoras para desarrollar el proceso de enseñanza - aprendizaje.

Por otra parte, el cambio de paradigma que emerge en el día a día por la implementación de las TIC en el aula y la adquisición de competencias en TIC por los docentes, conlleva a realizar una investigación sobre las estrategias de enseñanza con uso de TIC por parte del docente que favorezcan la convivencia escolar en los estudiantes y propicien elementos para el desarrollo de prácticas pedagógicas exitosas y vigentes con las políticas nacionales.

TIC como estrategia pedagógica para la convivencia en instituciones educativas

La aceleración del cambio tecnológico hace cada vez más caduco el estático planteamiento educativo tradicional. De la misma manera, la sociedad siente la inadecuación de los productos que proporcionan los sistemas educativos formales con semejanza a las necesidades del actual y futuro inmediato. Producto de ello es el deseo, a menudo no explícito, de una revisión a fondo de las estructuras modificando las de forma tal que permitan una mayor y más rápida adaptación.

A propósito, el desarrollo de nuevas instituciones, tecnologías y inclinación de viejos procesos llevan a la aparición de novedosas carreras profesionales, modos de producción y gestión, sugiriendo la es- casez de una educación permanente y continuada. Brevemente, los problemas que se intuyen en el futuro cercano radican, en buena medida, en la incapacidad de los sistemas educativos para anticiparse y acomodarse a los cambios sociales, los cuales hoy en día están condicionados, entre otros factores, por el cambio tecnológico, en este sentido, en educación el cambio se considera como la respuesta al inconveniente identificado.

Lo que quiere decir, que es evidente que los sistemas educativos del futuro deberán ser de una capacidad de adaptación muy preferente a la actual. La formación continua y diversificada será una de las fórmulas a adoptar. La promoción de nuevos productos y servicios de la tecnología de la información está ya ejerciendo un fuerte tirón en la demanda de personal capacitado para operar, gestionar, diseñar, construir y explotar los diversos elementos constituyentes de los sistemas de información y comunicación, en general, la educación actual aún no está preparada para asumir y conducir este cambio de rumbo. Así mismo, es preciso ir preparando una fuerza laboral e intelectual muy creativa y cualificada para abordar los problemas que, por la rápida evolución de la tecnología, no se podrán resolver a medio plazo si no se toman las medidas a tiempo.

Según diversos autores las tecnologías de la información y la comunicación, permiten desarrollar competencias sociales, que incluso se ha utilizado para trabajar en instituciones educativas con deterioro de la convivencia escolar como factores asociados al desempeño en estudiantes de básica primaria con experiencia de desplazamiento forzado (Avendaño, Cortés, Guerrero, 2015).

En efecto, la tecnología de la comunicación está perfectamente enraizada con 
la información dando lugar al desarrollo de las TIC. La cultura colectiva está cambiando substancialmente de estar soportada por los libros a estar basada en el ordenador a través de los medios y soportes de información y comunicación que conforman las TIC. Hasta ahora, y por otras razones, el efecto que se ha producido en la educación reglada (formal) ha sido más amortiguada que en otros tipos de formación.

Las TIC, en la educación se conciben como recursos tecnológicos y estrategias pedagógicas, innovadoras, que representan transformaciones en la metodología de enseñanza, propiciando espacios de interacción y comunicación entre docentes y estudiantes, debido a que la integración de las TIC implica generar cambios en los modelos pedagógicos tradicionales por otros que permitan desarrollar el trabajo a un nivel tecnológico orientado al aprendizaje del individuo.

Según Cacurri, (2013). Cada día aumentan la cantidad de docentes que utilizan herramientas informáticas, por ende, estas se conciben como el universo de dos conjuntos, representadas por las tradicionales Tecnologías de la Comunicación (TC), constituidas principalmente por la radio, televisión, telefonía convencional y Tecnologías de la información (TI) caracterizadas por la digitalización de las tecnologías de registros de contenidos sean estas (informática, comunicación, telemática e interfaces). Se puede notar que en las TIC se presentan dos vertientes un antes y un después, pero lo importante es que siempre han estado presentes en los procesos educativos, para mejorar la interacción en el proceso de enseñanzaaprendizaje.

Por otro lado, Herrera, (2016) menciona que las TIC se han convertido en una herramienta innovadora en el contexto educativo generando una relación ineludible que se puede aplicar a cualquier modelo pedagógico a desarrollar y a tener presente de forma trasversal como instrumento pedagógico.

Así mismo Cabero (2012), agrupa las TIC en tres sistemas de comunicación: video, informática y telecomunicación, los cuales abarcan los siguientes medios: videos interactivos, videotextos, teletextos, televisión por cable, páginas web, sistemas multimedia, teleconferencias, audio conferencia, videoconferencia, sistemas expertos: (realidad virtual, telemática y tele presencial), dando como resultado tres fases sistemáticas, las cuales dan lugar al compendio de avances que se proporciona al hombre en su medio social para dar solución a problemas de su entorno.

Partiendo de lo anterior se puede afirmar que las TIC, son un conjunto de procesos y productos derivados de las herramientas tecnológicas (hardware y software), como soportes de la información y canales de comunicación relacionados con el almacenamiento, procesamiento y transmisión digitalizada de la Información garantizando al hombre las herramientas necesarias para la solución de problemas. En tal sentido Marqués (2013), se refiere a las TIC como la aplicación de los conocimientos científicos para facilitar las actividades del hombre. Puesto que permite la creación de productos, instrumentos, lenguajes y métodos al servicio de las personas. Donde la información resulta fundamental para las personas.

En tal sentido las Tic producen diversidad de usos, por ello las mismas están clasificadas de acuerdo a las funciones que se deseen desempeñar, los usos pueden ser prácticos, pedagógicos e instructivos, produciendo procesos co- 
municacionales en la interacción entre emisor y receptor generando así una relación directa entre alumnos y docentes en la práctica pedagógica. Según Cabero (2012), en los últimos años las TIC han ganado un espacio importante en el campo educativo, donde las aplicaciones web y el desarrollo de las telecomunicaciones, surgen como una valiosa herramienta para la sistematización de los conocimientos que adquieren los estudiantes en su proceso de formación.

Por otro lado, los recursos informáticos son elementos disponibles para resolver una necesidad o aprendizaje con la aplicación de las TIC, estos impulsan los modelos de aplicación para los proyectos educativos fundamentalmente para el desarrollo de las actividades en el aula. Al respecto señala Coll (2012), Estos ofrecen la posibilidad de brindar al proceso educativo que cada alumno o docente elabore sus propios con códigos de pensar, sentir, o hacer propiciando el proceso de reconstrucción y desarrollo personal de recreación cultural.

A su vez los recursos informáticos integran softwares educativos que son programas pedagógicos didácticos los cuales sirven para trabajar de forma interactiva en los equipos de cómputo creados con la finalidad específica de ser utilizados como estrategias pedagógicas, es decir, para facilitar los procesos de enseñanza y de aprendizaje. Bartolomé, (2013). Expone que todos los softwares o programas de usos generales en el ámbito educativo poseen funciones didácticas como; enseñar ciencias, física, matemáticas entre otros, sin embargo, el docente deberá usar su ingenio para lograr una formación apoyada en la construcción de los nuevos conocimientos determinando la forma y momento oportuno para la integración en su práctica pedagógica.
Hablar de practica pedagógica implica que el docente diseñe estrategias para transmitir el conocimiento, donde debe planificar, evaluar, saber enseñar, saber dirigir grupos, entre otras actividades que se realizan en un contexto escolar. La escuela busca educar alumnos para la vida, para que se forme como un ciudadano crítico, analítico, constructivo, autónomo y solidario con capacidades de aprender a trabajar y así, resolver sus necesidades en el contexto social que viva.

Según Agullas (2014), la practica pedagógica es una labor educativa que desarrolla el docente de una forma creativa, en el sentido de trasformar el comportamiento de los educandos, dirigidos a un mecanismo de formación para la participación activa en la sociedad y este debe ser intencional o consiente. Esto quiere decir que educar es preparar al educando a que resuelva los problemas y las necesidades que surjan a su alrededor. No obstante, para que esto suceda el docente debe saber impartir una pedagogía de calidad al momento de trasmitir conocimientos.

En la actualidad las TIC han adquirido una importancia relevante en el proceso enseñanza aprendizaje. Se han convertido en el eje transversal de la acción formativa a través del conjunto de herramientas, soportes y canales que facilitan los procesos de aprendizaje, considerando elementos fundamentales como el "acceso a la información" y la "comunicación" en espacio y tiempo. El principal aporte de las TIC radica en el hecho de constituirse en un canal de comunicación inmediata. La a apropiación de las TIC por el docente es clave para la incorporación de estas al sistema educativo en la sociedad de la información y el conocimiento, ya que este debe estar acompañado de planes y programas que satisfagan el desarrollo 
integral de las comunidades con el uso o aplicación de las mismas en su práctica pedagógica.

En consecuencia, se hace menester definir las estrategias pedagógicas, estas son un sistema de acciones que se realizan con un ordenamiento lógico y coherente en función del cumplimiento de objetivos educativos. Es decir, constituye cualquier método o actividad planificada que mejore el aprendizaje profesional y facilite el crecimiento personal del estudiante. (Picardo, Balmore, \& Escobar, 2014) Por otro lado, se define como un conjunto de acciones que implican un proceso planificado con un propósito educativo, para la aplicación de unas herramientas y recursos que permiten acceder a un resultado significativo.

Por su parte, la convivencia escolar se entiende como un proceso de interrelación entre los diferentes miembros de un establecimiento educativo, según Good y Brophy (2012) "es el proceso por el cual un sujeto adquiere o desarrolla una nueva conciencia y conocimiento de su capacidad de aprendizaje, para adaptarse al ámbito escolar que le proporciona nuevos significados". Explican los autores antes señalados, que para que el aprendizaje sea posible, los intercambios entre todos los actores de la institución que comparten la actividad en la escuela y que conforman esa red de vínculos interpersonales que se denomina convivencia deben construirse cotidianamente, mantenerse y renovarse cada día, según determinados valores.

Para Good y Brophy (2012), "sólo cuando en una institución escolar se privilegia la comunicación, el respeto mutuo, el diálogo, la participación, recién entonces se genera el clima adecuado para posibilitar el aprendizaje y convivencia, pues, aunque parecen términos diferentes, están relacionados entre sí. La causalidad circular permite comprender la interrelación entre ambos, donde cada uno es condición necesaria para que se desarrolle el otro.

De acuerdo a Woolfolk (2010), el concepto de convivencia se refiere "al accionar de grupos sociales democráticos, a las relaciones entre sus miembros y las normas que estos comparten. En tanto estas normas surgen de una concertación entre partes, la convivencia es un proceso en permanente construcción en cada institución educativa que debe ser aceptado e internalizado por cada uno de sus miembros. Además, para darse la convivencia escolar se enuncian algunos principios que deben ser considerados por todos los integrantes de la institución escolar ya que el cumplimiento de las normas de convivencia por parte de todos es la garantía del mejoramiento de las relaciones no sólo de las interpersonales, sino también de las intersectoriales. Cada uno de los roles que se desempeñan en la escuela determina deberes y derechos diferenciados en la mayoría de las conductas.

Good y Brophy (2012), señalan que existen "tres ámbitos fundamentales que establecen el nivel de convivencia en una institución educativa, estos incluyen; comunicación, relaciones interpersonales y solución de conflictos"; abordar la convivencia en la escuela, como parte de la formación general e ineludible de los sujetos que aprenden, nos remite a la búsqueda participativa y deliberada de nuevos sentidos para la convivencia humana. Se de-be entender que innovar para mejorar la convivencia posibilita el desarrollo del nivel de la misma. Sin embargo, para Vergara (2010), "el aprendizaje profundo de la comunidad educativa es el que propicia el desarrollo de habilidades permanentes para visualizar participativamente las 
fortalezas, mejorando y reconstruyendo pedagógicamente el nivel de convivencia escolar", de esta manera, se estimulan aprendizajes de calidad en los niños, niñas y jóvenes del país.

Por su parte, Lagos (2009), menciona que es necesario "mejorar la convivencia social en las escuelas", esto significa asegurar relaciones respetuosas entre los miembros de la comunidad educativa y superar las prácticas discriminatorias. Significa enseñar a los estudiantes a desenvolverse en una institucionalidad formada por reglas de colaboración, resolviendo los conflictos de manera justa $\mathrm{y}$ formativa.

En este punto las relaciones interpersonales son un aspecto importante para mencionar, puesto que estas permiten el contacto y la interacción del ser humano en sociedad. En igual forma, Good y Brophy (2012), definen las relaciones humanas como "el trato o la comunicación que se establece entre dos o más personas". Sin embargo, para que se desarrolle el proceso de socialización son muy importantes las instituciones escolares, puesto que durante la actividad educativa se produce un proceso recíproco mediante el cual las personas que se ponen en contacto valoran los comportamientos delos otros y se forman opiniones acerca de ellos, todo lo cual suscita sentimientos que influyen en el tipo de relaciones que se establecen.

Texeidó y Capell (2012) indican que las relaciones interpersonales se dan cuando el individuo aprende a relacionarse con las demás personas respetando su espacio y aceptando sus diferencias, con defectos y virtudes, recordando que todo ser humano es sujeto de derechos, a su vez Chávez (2007), expresa que la relación interpersonal es una interacción recíproca entre dos o más personas. Pero estas al ser relaciones sociales se encuentran reguladas por leyes e instituciones, además, el mismo autor enfatiza que el análisis conjunto de estas relaciones, constituye una fase previa para identificar el clima social que se configura en el aula.

Para Gómez (2008), significa entonces que los procesos interpersonales al interior de los centros educativos y su relación con los resultados deseados son importantes para el estudio del ambiente o clima social escolar, puesto que, si las relaciones que se producen como consecuencia de la tarea educativa en común está cargado de interacciones socio-afectivas armoniosas, el clima de la clase será gratificante y contribuirá a crear condiciones favorables para el aprendizaje; por el contrario, si está caracterizado por la competencia, agresividad, envidia e intriga, el clima será poco gratificante y por consiguiente las condiciones para el proceso de aprendizaje serán poco favorables.

Dicho en otras palabras, la educación se puede considerar como un sistema que presenta una dualidad notoria: por un lado, es abierto e interactúa con un entorno social, político, cultural y económico, por otro se comporta como un sistema cerrado de bajo dinamismo, ya que dispone de elementos internos capaces de lograr sus objetivos y establecer esquemas de medición, evaluación, y corrección de sus resultados. Como sistema presenta elementos, flujos de información, entradas de recursos de transformación (humanos, financieros, de conocimiento) y salidas. Las tecnologías del aprendizaje representan una de las fuerzas renovadoras en los sistemas de aprendizaje y constituyen un elemento clave para el desarrollo de la educación y la formación.

En efecto, una de las finalidades de la educación es capacitar al alumnado para comprender, crear y participar en 
la cultura de su tiempo. Las TIC suponen en ese ámbito una nueva forma de organizar, representar y codificar la realidad, son además instrumentos valiosos para lograr un elevado grado de aplicación de los conocimientos adquiridos. El profesional de la enseñanza no puede permanecer ajeno a esta situación, por lo que debe hacer un esfuerzo en lo que a la actualización tecnológica se refiere una vez superada la posible intimidación que la tecnología puede suponer.

\section{Metodología}

\section{Diseño}

La metodología utilizada en el estudio es de tipo cualitativo, entendida como "una categoría de diseños de investigación que extraen descripciones a partir de observaciones que adoptan la forma de entrevistas, narraciones, notas de campo, grabaciones, transcripciones de audio, vídeo, registros escritos de todo tipo, fotografías o películas y artefactos. Pérez (2012). A su vez se utilizó el modelo de Investigación acción que es una metodología de investigación orientada a la práctica educativa cuyo propósito general es mejorar las prácticas educativas.

Las características principales de este modelo son la transformación y mejora de una realidad educativa, partiendo de la perspectiva de quien posee el problema, implica la colaboración de participantes, requiere la reflexión sistemática en la acción, convirtiendo la práctica en objeto de investigación, formando parte del mismo proceso, esta es realizada por las personas implicadas en la investigación, donde el elemento de "formación" es esencial en el proceso investigativo.

\section{Participantes}

La población beneficiaria con la presente investigación estuvo conformada por ciento veintiséis (126) estudiantes de la Institución Educativa Departamental Humberto Velásquez García, sede Cristo Rey, estos fueron seleccionados de manera intencional puesto que presentan características comunes ante el problema de investigación, en cuanto a la caracterización sociodemográfica, estos habitan en el corregimiento de Sevilla Zona Bananera, en grados cuarto y quinto de primaria, sus edades oscilan entre $8 \mathrm{y}$ 16 años, en su totalidad pertenecen a estratos económicos bajos.

\section{Técnicas e instrumentos}

En cuanto a los instrumentos de recolección de información, se utilizó la bitácora o diario de campo para que los estudiantes indagaran en sus medios familiares y comunidades, relatorías individuales, institucionales, observación directa, encuestas, información suministrada por los estudiantes y un mural titulado; soy niño soy paz. Así, la propuesta de investigación se estructuro como una apuesta pedagógica en la Institución Educativa Humberto Velásquez García, sede Cristo Rey, para determinar las relaciones y procesos multiculturales en un grupo de estudiantes.

\section{Resultados}

De los planteamientos se de deduce que, considerando las TIC como estrategia pedagógica para la convivencia en instituciones educativas, se conciben como una herramienta potente en cuanto a la posible información que ofrecen y con correlación a la capacidad de acción y de actuación que ejercen sobre los usuarios. 


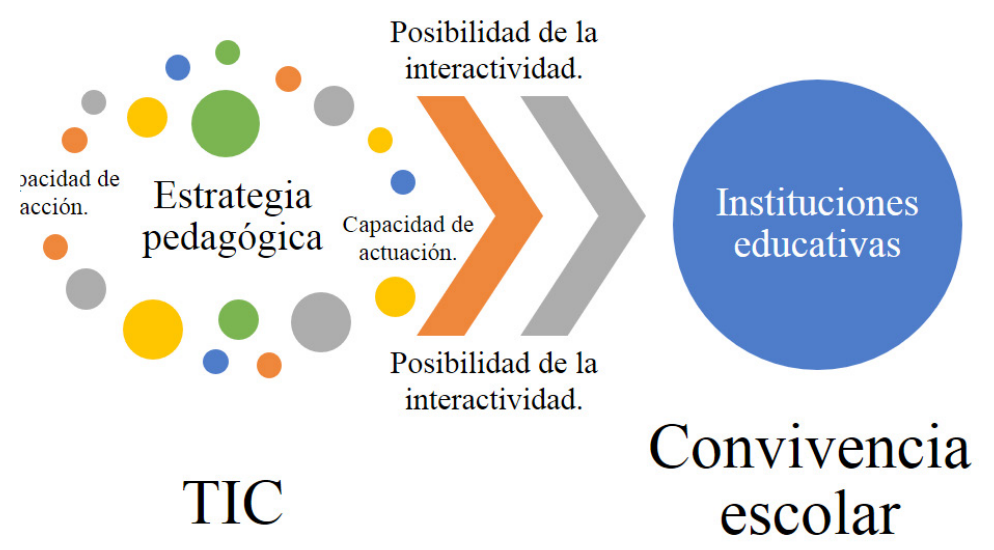
Medios y Recursos que se incorporan para desarrollar actividades, contenidos y objetivos educativos.

\begin{abstract}
Construcción de un modo de relación entre las personas de una comunidad.
\end{abstract}

Figura 1. Concepción de las TIC como estrategia pedagógica para la convivencia en instituciones educativas Fuente: elaboración propia (2018).

Presenta la posibilidad de la interactividad como fuente comunicativa entre individuos, objetos, conocimiento y maneras de procesar la información. Hay que señalar que un gran número de tecnologías ya se viene incorporando a actividades comunes, en el hogar y en el trabajo. En definitiva, son el fruto de una sociedad industrial, de la información y del conocimiento generado por ella que está suponiendo una profunda transformación en múltiples campos de la actividad humana escolar, (ver figura 1).

El estudio aplicado en la Institución Educativa Humberto Velásquez García, sede Cristo Rey permitió implementar las TIC como herramienta metodológica utilizada para generar cambios en las relaciones escolares de los alumnos, propiciando ambientes óptimos de convivencia pacífica dentro de la Institución, para determinar las relaciones y procesos multiculturales en el grupo de estudiantes, se diseñaron cuatro criterios y estrategias de comunicación apoyadas desde la exploración de escenarios interactivos, a través de las TIC:

En cuanto a los cuatro criterios se tuvo en cuenta, primero; que la experiencia fuera significativa, donde se crearon actividades en una plataforma virtual que respondieron a realidades culturales de los estudiantes. En segundo lugar, que fuera pertinente, lo cual implicó que las experiencias de las actividades propuestas lograran integrar las características y demandas del área de humanidades, con características particulares de la población estudiantil a la que iba dirigida, propiciando así un aprendizaje en contexto. Como tercer criterio se tuvo la innovación, es decir que la propuesta de mediación adecuara sus contenidos a las características de los estudiantes y a la propuesta comunicativa desde la innovación. Finalmente, como cuarto criterio, se contempló que la propuesta fuera generadora de aprendizajes, transferibles entre ellos, entre sus familias y como proceso reflexivo de sí mismos. 
Por su parte el diseño de las estrategias de comunicación, se plantearon como complemento para la plataforma virtual, incorporando a través del juego un modelo de reconstrucción social de la educación multicultural promoviendo el reconocimiento, auto concepto y relaciones sociales entre estudiantes de la Institución Educativa, lo anterior con la finalidad generar participación e interacción de los estudiantes en las actividades propuestas.

Seguidamente a través de la propuesta de mediación tecnológica, se plasmaron actividades realizadas por los estudiantes en un mural que se denominó "Soy niño soy paz", transformando espacios en la institución, con un modelo de reconstrucción social de la educación multicultural. La propuesta pedagógica planteada se desarrolló desde la construcción de un espacio web utilizando la herramienta blogger, como plataforma virtual donde reposaron las actividades desarrolladas por los estudiantes, además se creó una cuenta en YouTube como estrategia de divulgación de la información, estas se pueden observar en los siguientes enlaces; https://www.blogger.com/blogger. g?blogID=9101547403920200663\#allp osts https://www.youtube.com/channel/ UCZK7p51qYm7_LPyHrwi7Arg?view_ as=subscriber

\section{Conclusiones}

Dando respuesta al objetivo de la investigación, se concluye que mediante ambientes de aprendizaje apoyados con herramientas TIC se potencian los aprendizajes, formación ciudadana y convivencia escolar, sin embargo, esto requiere procesos continuos y pertinentes que obedezcan a la práctica y que promuevan actividades simuladas de la realidad para que los estudiantes desarrollen posturas y construyan propuestas de solución relevantes para su contexto social. Puesto que el dinamismo proporcionado por las TIC asigna nuevos roles en el aula, haciendo del aprendizaje un proceso autónomo, donde los docentes sean orientadores en el proceso presentando pautas para una educación responsable y así los estudiantes busquen comprender el mundo que los rodea interpretando y describiendo sus problemas, para luego hacer propuestas de mejora a las problemáticas presentadas en la misma.

En otras palabras, la aplicación de las TIC complicará las cosas y defraudará las expectativas si no va acompañada de una organización adecuada. Estas se deben cambiar tanto las actitudes como los métodos. Estos dos últimos elementos deben darse en el personal implicado para que se produzcan condiciones favorables que potencien la detección de factores restrictivos, la consolidación de juicios propios, la capacidad de anticiparse y prever con eficacia, y la mejora de la actitud para el razonamiento lógico. Como han demostrado diferentes estudios, el uso de las TIC en la educación depende de múltiples factores (infraestructuras, formación, actitudes, apoyo del equipo directivo, etc.), entre los cuales el más relevante es el interés y la formación por parte del profesorado, tanto a nivel instrumental como pedagógico.

Lo que quiere decir que, el impacto de las Tecnologías de la Información y Comunicación (TIC) sobre la educación, favorece uno de los mayores cambios en el ámbito de la Educación. A través de Internet y de las informaciones y recursos que ofrece, en el aula se abre una nueva ventana que nos permite acceder a múltiples recursos, informaciones y comunicarnos con otros, lo que nos ofrece la posibilidad 
de acceder con facilidad a conocer personalidades de opiniones diversas. Por otro lado, las nuevas teorías de aprendizaje que centran su atención no tanto en el profesor y el proceso de enseñanza, como en el alumno y el proceso de aprendizaje, tienen un buen aliado en estos medios, si se utilizan atendiendo a los postulados del aprendizaje socio constructivo y bajo los principios del aprendizaje significativo.

Con el uso de las TIC como estrategia pedagógica se pude lograr que los estudiantes se conviertan en ciudadanos informados y propositivos para su realidad social y a su vez adquieran una formación pertinente basada en la indagación, donde confronten la información y las intenciones de los diferentes autores para construir su propia opinión sobre los acontecimientos y sucesos sociales. Por tal motivo, los resultados de esta investigación sugieren una revisión urgente de los modelos pedagógicos empleados y del sistema educativo en su generalidad para lograr en los estudiantes aprendizaje significativo y activo y poder brindar a los egresados una educación flexible en tiempo, espacio, contenidos y ritmos de aprendizaje.

Por otro lado, los estudiantes adquieren un manejo responsable y ético sobre la información que reciben, procurando que esta sea constructiva y permita transformar su contexto en ambientes de bienestar. Así estos adquieren un compromiso con su propia formación, involucrándose de tal forma que desarrollen procesos formativos pertinentes y contextualizados que den sentido y transformen su entorno social. El manejo de conflictos entre los estudiantes requiere mecanismos de concertación y dialogo, que propongan soluciones a las diferentes dificultades en la interacción, promoviendo la convivencia pacífica.
La participación de estudiantes en escenarios democráticos permite que sean actores de cambio y transformación, promoviendo su intervención para convertirse en personas propositivas que aporten soluciones en sus contextos sociales, adquiriendo conciencia sobre cultura $\mathrm{y}$ diversidad, velando por derechos humanos, reconociendo valores individuales y sociales, aceptando diferentes creencias, ideologías y culturas, mediante la tolerancia y el respeto por los demás.

Finalmente, la implementación de las TIC fue de gran importancia en la institución, puesto que brindó dinamismo a la práctica docente, alternando en las diversas herramientas virtuales, para interactuar con los estudiantes, lo cual genera un aprendizaje significativo, donde existe mayor concentración, motivación y desarrollo de competencias cognitivas. En ese sentido, las TIC han sido una alternativa para mantener a los estudiantes ocupados en construir su conocimiento evitando generar conflictos en el aula, lo cual permite un desarrollo oportuno de la clase, favoreciendo el proceso enseñanzaaprendizaje.

\section{Referencias Bibliográficas}

Agullas, M. (2014). El Docente y los Desafíos de las TIC. Rev. Agenda Académica. Vol. 12, $N^{\circ}$ 1. Caracas.

Ávalos, G. (2008). El uso de la tecnología de la información y la comunicación y el diseño curricular. Revista Educación, 32 (1), 77-97.

Avendaño, I., Cortés, O., \& Guerrero, H, (2015). Competencias sociales y tecnologías de la información y la comunicación como factores asociados al desempeño en estudiantes de básica primaria con experiencia de desplazamiento forzado. Divers: Perspect. Psicol. Vol. 11. $N^{o} 1$. 
Bartolomé, A. (2013). Las Tecnologías de la Información y la Comunicación en la Escuela La Sociedad del Conocimiento $y$ de la información. España.

Cabero, J. (2012). El rol del profesor ante las nuevas tecnologías de la información y la comunicación. Rev. Agenda académica, 7(1), 41-57.

Cacurri, V. (2013). Revista Educación con TIC, nuevas formas de enseñar en la era digital. Buenos Aires.

Chávez. (2007). Introducción a la Investigación Educativa. Caracas - Venezuela

Coll. (2012). Revista para la difusión y el Uso educativo de las Tecnologías de la Información y la Comunicación.

Garrell, T. (2009). Aprender a convivir. Laboratorio Educativo. Caracas.

Gómez. (2008). Conflicto Comunicacionales en la administración escolar. Volumen 3. Trillas. México.

Good \& Brophy. (2012). Clima organizacional. La Muralla. Madrid.

Herrera-Tapias, B. (2016). Cultura Ciudadana y las Tecnologías de la Información y la Comunicación. Editorial YOYOBIZ. Barranquilla-Colombia.

Lagos. (2009). Valores. Editorial Santillana Caracas.

Márquez. (2013). Revista para la difusión y el Uso educativo de las Tecnologías de la Información y la Comunicación. Ministerio de Educación Caracas Venezuela. $\mathrm{N}^{\mathrm{o}} 01,02,05,08,09,11,13$. Disponible en: infofundabit@.me.gov.ve.
Pérez, G. (2012). Competencias o pensamiento práctico. La construcción de los significados de representación y de acción. Educar por competencias ¿qué hay de nuevo?. (2aed., pp. 59102). Madrid, España: Ediciones Morata.

Presidencia de la República de Colombia. (1991). Constitución política de Colombia. Recuperado de: http:// wsp.presidencia.gov.co/Normativa/ Documents/Constitucion-PoliticaColombia. Pdf.

Picardo Joao, O Balmore Pacheco, R., \& Escobar Baños, J.C. (2014). Diccionario enciclopédico de ciencias de la educación. San Salvador: El Salvador.

Teixidó S. y Capell C, D. (2012). Formación del profesorado orientada al desarrollo de competencias de gestión del aula de ESO. El afrontamiento de situaciones críticas. Disponible en Rev. Elect. Interuniv. Form. Profr., 5(1) (2012) (ISSN 1575-0965).

Vergara. (2010). La Cultura Organizacional en una Institución de Educación Superior. Tesis Doctoral. USB, Caracas - Venezuela.

Woolfolk. (2010). Diseño de la Organización. Legis, Santafé de Bogotá. 